\title{
Prevalence of Malnutrition among Patients on admission to a pediatric hospital.
}

\author{
Amira Aly Salem*, Mohamed Amr Hussein ${ }^{* *}$ and Lobna Monsour*. \\ *Pediatric Dept. Faculty of Medicine, Cairo University. \\ ${ }^{* * N}$ National Nutrition Institute.
}

\begin{abstract}
At the time of admission to the pediatric hospital of Cairo University 150 Children whose ager anged form birth to 12 years; 106 males and 44 females compiaining of acute or chronic medical illness, were examined to assess their nutrition status. The aim was to know the extent of malnutrition problem among them. Their weight and length/ height were obtained as well as blood hemoglobin, serum albumin and total lymphocyte count. Most of the patients were coming from families of low socio economic class. The study revealed high prevalence of malnutrition and anemia together with low blood lymphocytic count. This may have predisposed to their medical iliness. Hence, it is recommended to supply proper nutrition care to help limiting the period of hospital stay.
\end{abstract}

\section{Introduction}

Malnutrition is one of the leading causes of morbidity and mortality in childhood. It may result from improper or inadequate food intake or may result from inadequate absorption of food. Requirements of essential nutrients may be increased during stress and disease and the administration of antibiotics or of catabolic or anabolic drugs. The diagnosis of malnutrition rests on accurate dietary history and on evaluation of present deviations from average anthropometric measurements. Deficiencies of some nutrients may also be revealed by specific laboratory investigations.

Malnutrition usually involves deficits of more than a single nutrient. Immunologic insufficiency is common consequence of chronic malnutrition and can by demonstrated by total lymphocyte counts (Karp, 1993). The limited amount of documented information relating to nutrition at outpatient visits and on admission to hospital highlights the continued problem of lack of awareness of nutrition. Awareness of the importance of nutritional status by general practitioners 


\section{Amira Aly Salem, Mohamed Amr Hussein and Lobna Monsour .}

may afford interventions at an earlier stage and help in monitoring the efficacy of intervention (Bistarian et al \$976).

The aim of the study was to identify the major medical problems of children on admissions to the hospital and to test for evidence of any accompanying malnutrition.

\section{Paśient ard Methods:}

150 patients; 106 males and 44 females whose age ranged from birth to 12 years were admitted over a period of four months to Cairo University Children's hospital. On admission children were subjected to full history and clinical examination to diagnose the medical condition. All children were subjected to anthropometric measurements.

The full history taking included beside personal data, informations about socio economic status; this covered education and occupation of parents and household assets. Collected information were coded and classified into low, middle \& high socio economic classes, Also dietary history of the children were collected from the mothers which included feeding and weaning of infants.

Nutritional deficiency signs were examined according to Mclaren et al (1991). heights / Lengths, and weights were measured according to Jelliffe (1966). Laboratory tests to determine complete bood picture and serum albumiri itiere done before any medical intervention.

\section{Results:}

79 children out of the 150 were below 2 years and constitute $52.7 \%$ and $47.3 \%$ were above 2 years. The majority of cases (109) belong to families from low socio-economic class $(72.7 \%)$ and only $2 \%$ were of high socio-economic class.

Table 1 shows type for feeding of infants below 6 months where $60 \%$ were breast fed, $11.5 \%$ were bottle fed and $28.7 \%$ were breast $\&$ bottle feo.

Table 2 demonstrates the distribution of patients 6-12 months (28 cases) according to type food eaten. The table shows that children were given either breast, bottle or both, beside the weaning foods which is mostly cereals and of plant origin.

Table 3\&4 show the distribution of children (1-2 years and 2-12 years) respectively who consume different types of foods. Those who consume milk frequently or infrequently exceeded $70 \%$. The consumption of animal protein other than milk decreased in the age group 1-2 years 


\section{Egyptian J. of Nutrition and Health Vol. 1 No. 1 (2006)}

$11.7 \%$, to increase in the elder group $21.4 \%$. However those who consume eggs in the age group 1-2 years show litile increase (29.3\%) and high increase in the elder group (45.7\%).

Table 5 shows the prevalence of positive malnutrition signs among admitted children. It varied from high prevalence; teeth caries $39.3 \%$, lack of hair lustre $38.7 \%$ skin xerosis $32.7 \%$, spine deformity $26 \%$ rickets $25.3 \%$ edema of limbs $16.7 \%$; to low prevalence; face edema $2.7 \%$ and nail spooning $2.6 \%$.

Table 6 shows the prevalence of undernutrition by percentiles of weight for age and length or Height for age using the National Center of Health Statistics (NCHS) (Hamill 1979). 58\% of patients were below the $5^{\text {th }}$ percentile of weight for age and none were above the $95^{\text {th }}$ percentile. It also shows that $72.2 \%$ of children $<2$ years had severe or mild undernutrition compared with $83 \%$ of children 2-12 years. The prevalence of severe stunting (chronic undernutrition) was $68.7 \%$ and mild type was $20 \%$. Severe stunting is higher above 2 years of age compared with less than 2 years.

Table 7 shows the prevalence of acute PEM based on Waterlow (1974) (weight for height). The heighest prevalence of ccute PEM (all degrees) fell in the age group 2-4 years (35.1\%), followed by age less than 2 years (22.8\%). Severe mainutrition is higher among children less than 2 years $3.8 \%$ and no severe cases above 4 years. However, no acute PEM is recorded in the age group 6-8 years.

Table 8 shows the prevalence of chronic PEM based on waterlow criteria (1972) (height for age). Among the total cases $21.3 \%$ had severe stunting while the moderate and mild were $15.3 \%$ and $45.3 \%$ respectively. The highest percent of stunting was in the age group 8-12 years (100\%) followed by age group 4-6 years (94.4\%) Severe wasting is more prevalent among the younger two age groups.

Table $9 \& 10$ show the prevalence of acute and chronic PEM respectively in different acute diseases. Neonatal sepsis had the highest prevalence of acute PEM $44.4 \%$, followed by pneumonia cases (34.9\%) Neonatal jaundice, neonatal convulsions and Guillain Barre case did not show signs of acute PEM All cases of meningitis had mild chronic PEM, followed by pneumonia. $85 \%$ had all degrees of chronic PEM, then neonatal sepsis.

Table 11\&12 show the prevalence of acute and chronic PEM respectively in different chronic diseases. Cystic fibrosis had the highest prevalence of acute PEM followed by chronic 


\section{Amira Aly Salem, Mohamed Amr Hussein and Lobna Monsour .}

gastroenteritis . All cases of cystic fibrosis, cerebral palsy, rheumatic heart disease and thalassemia major had chronic PEM, while in chronic gastroenteritis $96.2 \%$ had chronic PEM.

Table 13 shows the overall percentage of anemia among children was 50.6 However anemia is more prevalent among children below 6 years $(56 \%)$, while it was $5.5 \%$ among those over 6 years. Those who had very low serum albumin $2.5 \mathrm{gm} \%$ constitute $23.4 \%$ and only $21.4 \%$ had serum albumin above $3.5 \mathrm{gm} \%$.

Table 14 shows the frequency and percent distribution of patients according to total lymphocyte count. Those who have count less than 500 cell $/ \mathrm{mm}^{3}$ were 34 patients $(22.6 \%), 25$ patients $(16.6 \%)$ had lymphocytes count between $500-1000$ cells $/ \mathrm{mm}^{3}$. Another 50 children $33.3 \%$ had count between $1000-1500$ and 41 Patients $27.5 \%$ had level above 1500 cells $/ \mathrm{mm}^{3}$.

\section{Discussion:}

Nutrition is fundamental to optimal rate of growth and development. The nutritional status of an individual has been shown to have important effects on health in recovery from: illness or injury (Mc Whirter and Pennington 1994).

We identified the prevalence of malnutrition on admission to hospital in patients with a wide variety of disease states as judged by anthropometric and laboratory data.

Of one hundred and fifty patients admitted to the general medical wards of Cairo University Children's Hospital over a period of 4 months (106 males and 44 females, ages ranging from birth to 12 years), the majority of admission $60 \%$ were for chronic conditions and $40 \%$ were for acute conditions.

Concerning weight for age; in our study $58 \%$ of patient were below the $5^{\text {th }}$ percentile, much higher than that of Kristy et al (1995) who found that only $16 \%$ of their patients were below the $5^{\text {th }}$ percentile None of our patient were above $95^{\text {th }}$ percentile compared with $7 \%$ in Kristy et al study according to the growth charts of the National Center of Health statistics (NCHS) Similar trend was found in respect to height where $78.7 \%$ of our patients fell below the $5^{\text {th }}$ percentile and none above $95^{\text {th }}$ percentile compared to $23 \%$ and $6 \%$ respectively among Kristy et al study (1995). The same authors demonstrated approximately the same prevalence of acute PEM as we did in our study, however, the moderate and severe degrees of malnutrition were higher in our study.

Merritt and Suskind (1979) found in their study that nearly half of their hospitalized pediatric patients $(47 \%)$ had chronic malnutrition which is less than what we noted in our study. We noticed 
that the prevalence of acute PEM in acute diseases was $23.3 \%$ and chronic PEM was present in $66.7 \%$ of cases. While prevalence of acute PEM in chronic diseases was $24.4 \%$ and chronic PEM was present in $92.2 \%$. There was no much difference in prevalence of acute PEM in acute and chronic diseases admitted but there is greater difference in prevalence of chronic PEM in chronic diseases.

The study conducted in Ghana by Conmey et al (1985) demonstrated that $67 \%$ of their patients were malnourished, classified into $54.3 \%$ having first degree, $32.3 \%$ and $13.4 \%$ had $2^{\text {nd }}$ and third degrees malnutrition. These cases were closely related to high incidence of malnutrition associated illness: diarrhea (74\%) measles (51\%) and intestinal parasites (54\%).

The majority of our patients came from low socio- economic class $(72.7 \%)$ and none of them complains of being malnourished. Commeys et al (1985) also stated that the majority of their patients came from low socio- economic households and mostly illiterate mothers. This illustrates that poor families are liable to have malnourished children that make them more susceptible to general medical diseases.

Prevalence of anemia $(\mathrm{Hb}<10 \mathrm{~g} \%)$ in $50.6 \%$ of our patients also supports our assumption. This figure is higher than that reported by Kristy et al (1995). Serum albumin values below $3.5 \mathrm{gm} \%$ were present in little more than half of cases reported by Kristy et al. while in our cases $76 \%$ had serum albumin less than $3.5 \mathrm{gm} \%$. Whitehead ef al (1973) regarded serum albumin as a good indicator of the pathophysiological changes noted with severe protein mainutrition. Bistarian ot al (1976) demonstrated significant relationship between malnutrition as judged by arm circumference and serum albumin. However, serum albumin level falls in response to infections or other stresses that trigger an acute phase response and therefore may not represent nutritional status perse. For instance, children having cancer may have low serum albumin levels that correlate more with fever than with decreased energy or protein dietary intake (Merritt et al 1980) Nonethless, hypolbuminemia is clearly an excellent marker for metabolic stress and multiple studies have correlated low serum albumin levels with mortality (Phillips et al, 1989) and length of stay (Herrman et al, 1992).

Our results demonstrated that $72.5 \%$ of the children had total lymphocytes less than $1500 / \mathrm{mm}^{3}$. This was higher than that reported by Kristy et al (1995) and Merritt and Suskind (1979). One of the most important consequences of nutritional depletion is depression of host resistance, especially cell mediated immunity (Keusch 1990) Immune competence is dependent 


\section{Amira Aly Salem, Mohamed Amr Hussein and Lobna Monsour .}

both on lymphocyte number and function. Cunningham- Rundles and Cervia (1997) have demonstrated the relationship between nutritional depletion and depressed immune function.

In conclusion we recognize from this study that prevalence of malnutrition in pediatric patients was high whether admitted for acute or chronic medical sickness. This denotes that growth failure may be attributed to mainutrition rather than disease. Hence, appropriate nutritional support of high risk hospitalized patients may be expected to improve their subsequent hospital course.

Table (1): Type of feeding and percent of infants $<6$ months using it ( $n=35$ cases).

\begin{tabular}{|l|l|l|l|l|}
\hline & Breast & Bottle & Breast \& Bottle & Total \\
\hline Frequency & 21 & 4 & 10 & 3.5 \\
\hline Percent & $60 \%$ & $11.5 \%$ & $28.5 \%$ & $100 \%$ \\
\hline
\end{tabular}

Table (2): Types of food eaten \& percent of infants (6-12 months) using it ( $n=28$ cases).

\begin{tabular}{|c|c|c|c|c|c|c|c|c|c|c|c|c|c|c|c|}
\hline \multicolumn{3}{|c|}{ Breast or Bottle milk } & \multicolumn{3}{|c|}{$\begin{array}{l}\text { Animal Protein } \\
\text { sources }\end{array}$} & \multicolumn{5}{|c|}{ Plant Protein sources } & \multicolumn{4}{|c|}{ Vegetables \& Fruits } & \multirow{3}{*}{ 愛 } \\
\hline 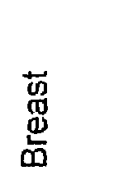 & $\begin{array}{l}\frac{\mathscr{U}}{\mathrm{z}} \\
\stackrel{0}{\mathrm{O}}\end{array}$ & 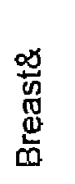 & 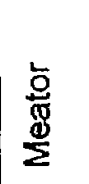 & 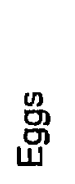 & & 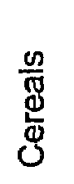 & & 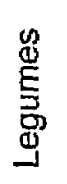 & $\frac{\mathfrak{d}}{\frac{\mathbb{x}}{\Sigma}}$ & $\begin{array}{l}\stackrel{0}{0} \\
\stackrel{0}{2}\end{array}$ & $\begin{array}{l}\frac{\Phi}{D} \\
\text { 焉 } \\
\text { D } \\
\text { > }\end{array}$ & 旁 & 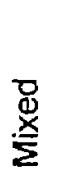 & $\frac{\mathscr{L}}{\mathrm{L}}$ & \\
\hline & & & Yes & No & Yes & No & Yes & & & & & & & & \\
\hline 14 & 6 & 8 & 6 & 22 & 8 & 20 & 3 & 5 & 4 & 16 & 5 & 5 & 8 & 10 & 28 \\
\hline $50 \%$ & $\begin{array}{l}21 . \\
4 \%\end{array}$ & $\begin{array}{l}28 . \\
5 \%\end{array}$ & $\begin{array}{l}21 \\
4 \%\end{array}$ & $\begin{array}{l}78 . \\
6 \%\end{array}$ & $\begin{array}{l}28 . \\
5 \%\end{array}$ & $\begin{array}{l}71 . \\
5 \%\end{array}$ & $\begin{array}{l}10 . \\
7 \%\end{array}$ & $\begin{array}{l}17 . \\
8 \%\end{array}$ & $\begin{array}{l}14 . \\
2 \%\end{array}$ & $\begin{array}{l}57 . \\
1 \%\end{array}$ & $\begin{array}{l}17 . \\
8 \%\end{array}$ & $\begin{array}{l}17 . \\
8 \%\end{array}$ & $\begin{array}{l}28 . \\
5 \%\end{array}$ & $\begin{array}{l}35 . \\
7 \%\end{array}$ & $\begin{array}{l}100 \\
\%\end{array}$ \\
\hline
\end{tabular}


Egyptian J. of Nutrition and Health Vol. 1 No. 1 (2006)

Table (3): Types of food eaten \& percent of Children ( $1-2$ years) using it ( $n=17$ cases).

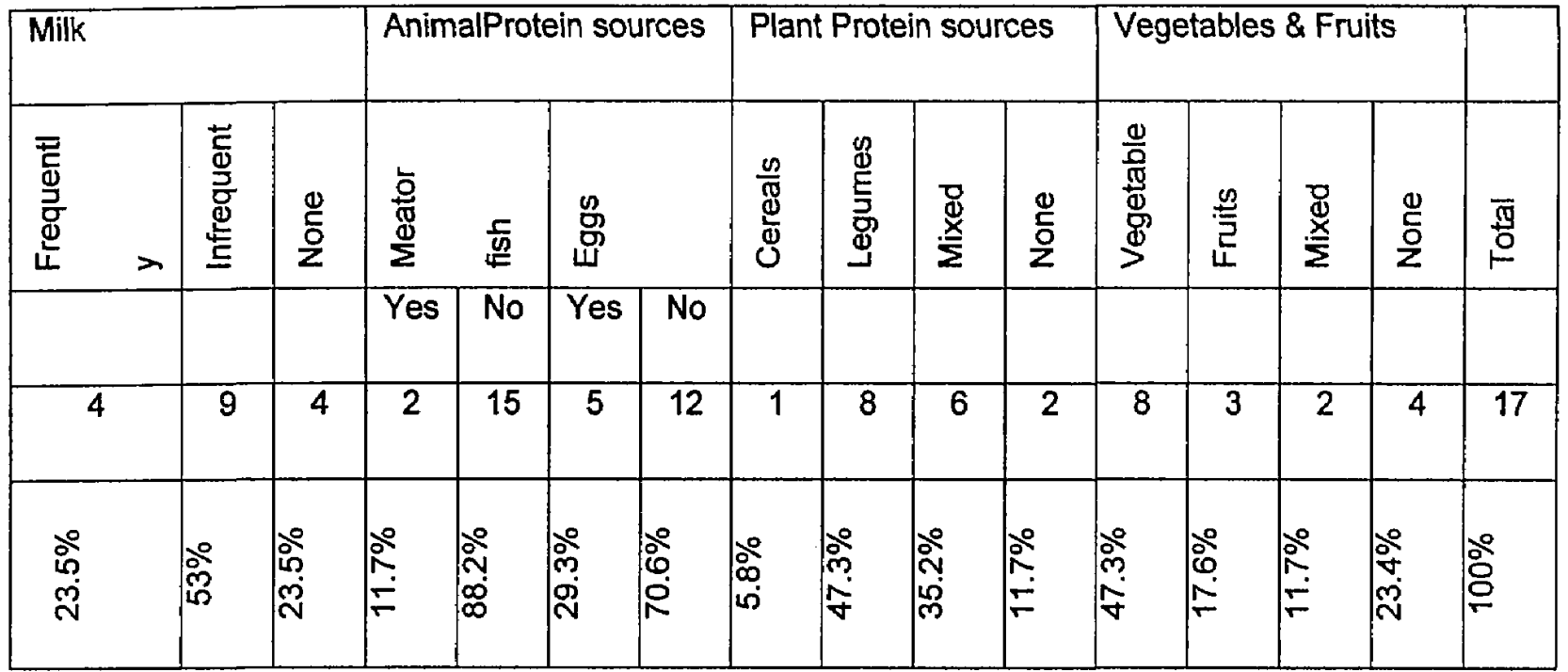

Table (4): Types of food eaten \& percent of Children (2-12 years) using it ( $n=70$ cases).

\begin{tabular}{|c|c|c|c|c|c|c|c|c|c|c|c|c|c|c|c|}
\hline \multicolumn{3}{|c|}{ Milk } & \multicolumn{4}{|c|}{ Animal Protein sources } & \multicolumn{4}{|c|}{ Plant Proteln sources } & \multicolumn{4}{|c|}{ Vegetables \& Frults } & \multirow{2}{*}{ 営 } \\
\hline \multirow{2}{*}{ 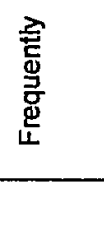 } & \multirow{2}{*}{ 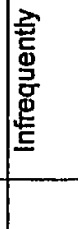 } & \multirow{2}{*}{ 总 } & \multicolumn{2}{|c|}{ Meat or fish } & \multicolumn{2}{|c|}{ Eggs } & \multirow{2}{*}{\begin{tabular}{|l}
$\frac{m}{8}$ \\
$\frac{8}{8}$ \\
$\mathcal{8}$
\end{tabular}} & \multirow{2}{*}{ 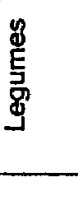 } & \multirow{2}{*}{\begin{tabular}{|l}
$\frac{8}{d}$ \\
$\frac{y}{z}$ \\
$z$
\end{tabular}} & \multirow{2}{*}{ 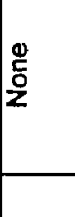 } & \multirow{2}{*}{ 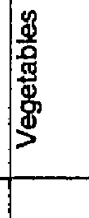 } & \multirow{2}{*}{ 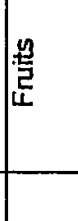 } & \multirow{2}{*}{ 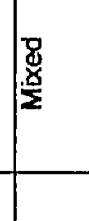 } & \multirow{2}{*}{ 哀 } & \\
\hline & & & Yes & No & Yes & No & & & & & & & & & \\
\hline 14 & 36 & 20 & 15 & 55 & 32 & 38 & 5 & 15 & 22 & 28 & 15 & 6 & 18 & 31 & 17 \\
\hline $20 \%$ & & 28.5 & 21.4 & 78.6 & 45.7 & 54.3 & 7.2 & 21.5 & 31.3 & $40 \%$ & 21.5 & 8.5 & 25.7 & 44.3 & 100 \\
\hline & $\%$ & $\%$ & $\%$ & $\%$ & $\%$ & $\%$ & $\%$ & $\%$ & $\%$ & & $\%$ & $\%$ & $\%$ & $\%$ & $\%$ \\
\hline
\end{tabular}




\section{Amira Aly Salem, Mohamed Amr Hussein and Lobna Monsour .}

Table (5): Prevalence of positive malnutrition signs (total 150 Cases).

\begin{tabular}{|l|l|l|l|}
\hline \multicolumn{2}{|l|}{ Sign } & No. & $\%$ \\
\hline Hair: & lack of lusire & 58 & 38.7 \\
\hline & Thinness \& sparseness & 12 & 8.0 \\
\cline { 2 - 4 } & Easy pluckability & 13 & 8.7 \\
\hline Face: & wasting & 36 & 24.0 \\
\hline & edema & 4 & 2.7 \\
\cline { 2 - 4 } & Parotid enlagement & 12 & 8.0 \\
\cline { 2 - 4 } & Lips angular stomatitis & 18 & 12.0 \\
\cline { 2 - 4 } & cheilosis & 19 & 12.7 \\
\hline Tongue glossitis & 18 & 12.0 \\
\hline Teeth caries & 59 & 39.3 \\
\hline Skin Xeroris & 49 & 32.7 \\
\hline Dermatitis & 20 & 13.3 \\
\hline Nails spooning & 4 & 2.6 \\
\hline Blepharitis & 57 & 38.0 \\
\hline Spine deformity & 39 & 26.0 \\
\hline Rachitic signs & 38 & 25.3 \\
\hline Edema of limbs & 25 & 16.7 \\
\hline
\end{tabular}

Table (6): Prevalence of underweight for age and prevalence of stunting using percentiles (total 150 Cases).

\begin{tabular}{|c|c|c|c|c|c|c|c|c|c|}
\hline \multicolumn{10}{|c|}{ Weight for age } \\
\hline \multirow{2}{*}{$\begin{array}{l}\text { Status of } \\
\text { malnutrition }\end{array}$} & \multicolumn{3}{|c|}{$<2 Y(N 79)$} & \multicolumn{3}{|c|}{$2-12 Y(N 71)$} & \multicolumn{3}{|c|}{$\%$ of total (N150) } \\
\hline & \multicolumn{2}{|l|}{$\mathbf{N}$} & $\%$ & \multicolumn{2}{|l|}{$\bar{N}$} & $\%$ & \multicolumn{2}{|l|}{$\bar{N}$} & $\%$ \\
\hline$<5^{\text {th }}$ severe & \multicolumn{2}{|l|}{45} & 56.9 & \multicolumn{2}{|l|}{42} & 59.1 & \multicolumn{2}{|l|}{87} & 58 \\
\hline $6^{\text {th }}-24^{\text {th }}$ mild & \multicolumn{2}{|l|}{12} & 15.3 & \multicolumn{2}{|l|}{17} & 23.9 & \multicolumn{2}{|l|}{29} & 19.3 \\
\hline \multirow[t]{3}{*}{$25^{\text {th }}-94^{\text {th }}$ None } & \multicolumn{2}{|l|}{22} & 22.8 & \multicolumn{2}{|l|}{$\overline{12}$} & 17 & \multicolumn{2}{|l|}{34} & 22.7 \\
\hline & \multicolumn{3}{|c|}{ Length for age } & \multicolumn{3}{|c|}{ Ht for age } & \multicolumn{3}{|c|}{$\%$ of total } \\
\hline & $\mathbf{N}$ & \multicolumn{2}{|l|}{$\%$} & $\mathbf{N}$ & \multicolumn{2}{|l|}{$\%$} & $\bar{N}$ & \multicolumn{2}{|l|}{$\%$} \\
\hline$<5^{\text {th }}$ severe & 53 & \multicolumn{2}{|l|}{67.0} & 50 & \multicolumn{2}{|l|}{70.4} & 103 & \multicolumn{2}{|l|}{68.7} \\
\hline $6^{\text {th }}-24^{\text {th }}$ mild & 14 & \multicolumn{2}{|l|}{17.7} & 16 & \multicolumn{2}{|l|}{22.5} & 30 & \multicolumn{2}{|l|}{20.0} \\
\hline $25^{\text {th }}-94^{\text {th }}$ None & 12 & \multicolumn{2}{|l|}{15.3} & 5 & \multicolumn{2}{|l|}{7.1} & 17 & \multicolumn{2}{|l|}{11.7} \\
\hline
\end{tabular}


Egyptian J. of Nutrition and Health Vol. 1 No. 1 (2006)

Table (7): Prevalence of acute PEM based on Waterlow (weight for height).

\begin{tabular}{|c|c|c|c|c|c|c|}
\hline \multicolumn{2}{|c|}{ Variable } & \multicolumn{5}{|c|}{ Acute PEM (wasting) Percent of the median } \\
\hline $\begin{array}{l}\text { Age } \\
\text { years }\end{array}$ & $\begin{array}{l}\text { Number \& \% of } \\
\text { malnutrition }\end{array}$ & $\begin{array}{l}\text { Severe } \\
<70\end{array}$ & $\begin{array}{l}\text { Moderate } 70 \text { - } \\
80\end{array}$ & Mild $81-90$ & None $>90$ & Total \\
\hline$<2$ & $\begin{array}{l}\text { Number } \\
\%\end{array}$ & $\begin{array}{l}3 \\
3.8 \%\end{array}$ & $\begin{array}{l}8 \\
10.1 \%\end{array}$ & $\begin{array}{l}7 \\
8.9 \%\end{array}$ & $\begin{array}{l}61 \\
77.2 \%\end{array}$ & $\begin{array}{l}79 \\
100 \%\end{array}$ \\
\hline $2-4$ & $\begin{array}{l}\text { Number } \\
\%\end{array}$ & $\begin{array}{l}1 \\
2.7 \%\end{array}$ & $\begin{array}{l}4 \\
10.8 \%\end{array}$ & $\begin{array}{l}8 \\
21.6 \%\end{array}$ & $\begin{array}{l}24 \\
64.9 \%\end{array}$ & $\begin{array}{l}37 \\
100 \%\end{array}$ \\
\hline $4-6$ & $\begin{array}{l}\text { Number } \\
\%\end{array}$ & 0 & $\begin{array}{l}1 \\
5.6 \%\end{array}$ & $\begin{array}{l}3 \\
16.7 \%\end{array}$ & $\begin{array}{l}14 \\
77.8 \%\end{array}$ & $\begin{array}{l}18 \\
100 \%\end{array}$ \\
\hline $6-8$ & $\begin{array}{l}\text { Number } \\
\%\end{array}$ & 0 & 0 & 0 & $\begin{array}{l}5 \\
100 \%\end{array}$ & $\begin{array}{l}5 \\
100 \%\end{array}$ \\
\hline $8-12$ & $\begin{array}{l}\text { Number } \\
\%\end{array}$ & 0 & 0 & $\begin{array}{l}1 \\
9.1 \%\end{array}$ & $\begin{array}{l}10 \\
90.9 \%\end{array}$ & $\begin{array}{l}11 \\
100 \%\end{array}$ \\
\hline Total & $\begin{array}{l}\text { Number } \\
\%\end{array}$ & $\begin{array}{l}4 \\
2.7 \%\end{array}$ & $\begin{array}{l}13 \\
8.7 \%\end{array}$ & $\begin{array}{l}19 \\
12.7 \%\end{array}$ & $\begin{array}{l}114 \\
76 \%\end{array}$ & $\begin{array}{l}150 \\
100 \%\end{array}$ \\
\hline
\end{tabular}

Table (8): Prevalence of chronic PEM based on Waterlow (height for age).

\begin{tabular}{|l|l|l|l|l|l|l|}
\hline \multicolumn{2}{|l|}{ Variable } & \multicolumn{5}{l|}{ Chronic PEM (stunting) Percent of the median } \\
\hline $\begin{array}{l}\text { Age } \\
\text { years }\end{array}$ & $\begin{array}{l}\text { Number \&\% of } \\
\text { malnutrition }\end{array}$ & $\begin{array}{l}\text { Severe } \\
<85\end{array}$ & $\begin{array}{l}\text { Moderate 85- } \\
89\end{array}$ & $\begin{array}{l}\text { Mild } \\
95\end{array}$ & $\begin{array}{l}\text { None } \\
>95\end{array}$ & Total \\
\hline$<2$ & $\begin{array}{l}\text { Number } \\
\%\end{array}$ & 17 & 18 & 24 & 20 & 79 \\
& $21.5 \%$ & $22.8 \%$ & $30.4 \%$ & $25.3 \%$ & $100 \%$ \\
\hline $2-4$ & $\begin{array}{l}\text { Number } \\
\%\end{array}$ & 14 & $2.7 \%$ & 18 & 4 & 37 \\
& $37.8 \%$ & $4.3 \%$ & $48.6 \%$ & $10.8 \%$ & $100 \%$ \\
\hline $4-6$ & Number & 1 & 1 & 15 & 1 & 18 \\
& $\%$ & $5.6 \%$ & $5.6 \%$ & $83.3 \%$ & $5.6 \%$ & $100 \%$ \\
\hline $6-8$ & Number & 0 & 1 & 2 & 2 & 5 \\
& $\%$ & & $20 \%$ & $40 \%$ & $40 \%$ & $100 \%$ \\
\hline $8-12$ & Number & 0 & 2 & 9 & 0 & 11 \\
& $\%$ & & $18.2 \%$ & $81.8 \%$ & & $100 \%$ \\
\hline Total & Number & 32 & 23 & 68 & 27 & 150 \\
& $\%$ & $21.3 \%$ & $15.3 \%$ & $45.3 \%$ & $18 \%$ & $100 \%$ \\
\hline
\end{tabular}


Amira Aly Salem, Mohamed Amr Hussein and Lobna Monsour .

Table (9): Prevalence of acute PEM in different acute diseases.

\begin{tabular}{|l|l|l|l|l|l|}
\hline Diseases & Severe $<70$ & Moderate 70-80 & Mild 81-90 & None $>90$ & Total \\
\hline Acute GE & 0 & 1 & 3 & 10 & 14 \\
& & $7.1 \%$ & $21.4 \%$ & $71.4 \%$ & $23.3 \%$ \\
\hline Guillain Barre & 0 & 0 & 0 & 1 & 1 \\
& & & $100 \%$ & $1.6 \%$ \\
\hline Meningitis & 0 & 0 & 1 & 3 & 4 \\
& & & $25 \%$ & $75 \%$ & $6.6 \%$ \\
\hline Neonatal Jaundice & 0 & 0 & 0 & 5 & 5 \\
& & & & $100 \%$ & $8.3 \%$ \\
\hline Neonatal Convulsion & 0 & 0 & 0 & 7 & 7 \\
& & & & $100 \%$ & $11.6 \%$ \\
\hline Neonatal sepsis & 1 & 3 & 5 & $55.6 \%$ & $15.3 \%$ \\
\hline Pneumonia & $11.1 \%$ & $33.3 \%$ & 1 & 15 & 20 \\
& 0 & $5 \%$ & $29.9 \%$ & $75 \%$ & $33.3 \%$ \\
\hline Total & & 5 & 8 & 46 & 60 \\
& 1 & $8.4 \%$ & $13.3 \%$ & $76.7 \%$ & $100 \%$ \\
\hline
\end{tabular}

Table (10): Prevalence of chronic PEM in different acute diseases.

\begin{tabular}{|l|l|l|l|l|l|}
\hline & Severe <85 & Moderate 85-89 & Mild 90-95 & None >95 & Total \\
\hline Acute GE & 1 & 1 & 6 & 6 & 14 \\
& $7.1 \%$ & $7.1 \%$ & $42.9 \%$ & $42.9 \%$ & $23.3 \%$ \\
\hline Guillain Barre & 0 & 0 & 0 & 1 & 1 \\
& & & & $100 \%$ & $1.6 \%$ \\
\hline Meningitis & 0 & 0 & 4 & 0 & 4 \\
& & & $100 \%$ & & $6.6 \%$ \\
\hline Neonatal Jaundice & 0 & 0 & 2 & 3 & 5 \\
& & & $40 \%$ & $60 \%$ & $8.3 \%$ \\
\hline Neonatal Convulsion & 0 & 3 & 0 & 4 & 7 \\
& & $42.9 \%$ & $57.1 \%$ & $11.6 \%$ \\
\hline Neonatal sepsis & 1 & 0 & 5 & 3 & 9 \\
& $11.1 \%$ & & $55.6 \%$ & $33.3 \%$ & $15.3 \%$ \\
\hline Pneumonia & 2 & 2 & 13 & 3 & 20 \\
& $10 \%$ & $10 \%$ & $65 \%$ & $15 \%$ & $33.3 \%$ \\
\hline Total & 4 & 6 & 30 & 20 & 60 \\
& $6.6 \%$ & $10 \%$ & $50 \%$ & $33.3 \%$ & $100 \%$ \\
\hline
\end{tabular}


Egyptian J. of Nutrition and Health Vol. 1 No. 1 (2006)

Table (11): Prevalence of acute PEM in different chronic diseases.

\begin{tabular}{|c|c|c|c|c|c|}
\hline Diseases & Severe $<70$ & Moderate 70-80 & Mild 81-90 & None $>90$ & Total \\
\hline Chronic GE & 3 & 3 & 1 & 19 & 26 \\
& $11.5 \%$ & $11.5 \%$ & $3.8 \%$ & $73.2 \%$ & $28.8 \%$ \\
\hline Cystic fibrosis & 0 & 5 & 2 & 0 & 7 \\
\hline Congenital H.D & 0 & 0 & $28.6 \%$ & $7.7 \%$ \\
\hline $\begin{array}{c}\text { Cerebral } \\
\text { palsy }\end{array}$ & 0 & 0 & 0 & 16 & 21 \\
\hline Renal failure & 0 & 0 & $1.4 \%$ & 4 & $23.8 \%$ \\
\hline Rh.H.D & 0 & 0 & 1 & $100 \%$ & $4.4 \%$ \\
\hline Sickle cell anaemia & 0 & 0 & 0 & 10 & 12 \\
\hline Thalassemia major & 0 & 0 & 0 & $7.3 \%$ & $13.33 \%$ \\
\hline Total & 3 & 8 & 11 & 7 & 8 \\
\hline
\end{tabular}

Table (12): Prevalence of chronic PEM in different chronic diseases.

\begin{tabular}{|c|c|c|c|c|c|}
\hline Diseases & Severe $<85$ & Moderate $85-89$ & Mild 90-95 & None $>95$ & Total \\
\hline Chronic GE & $\begin{array}{c}19 \\
73.2 \%\end{array}$ & $\begin{array}{c}5 \\
19.2 \%\end{array}$ & $\begin{array}{c}1 \\
3.8 \%\end{array}$ & $\begin{array}{c}1 \\
3.8 \%\end{array}$ & $\begin{array}{c}26 \\
28.8 \%\end{array}$ \\
\hline $\begin{array}{l}\text { Cystic } \\
\text { fibrosis }\end{array}$ & 0 & $\begin{array}{c}2 \\
28.6 \%\end{array}$ & $\begin{array}{c}5 \\
71.4 \%\end{array}$ & 0 & $\begin{array}{c}7 \\
7.7 \%\end{array}$ \\
\hline $\begin{array}{c}\text { Congenital } \\
\text { H.D }\end{array}$ & $\begin{array}{c}8 \\
38.1 \%\end{array}$ & $\begin{array}{c}7 \\
33.3 \%\end{array}$ & $\begin{array}{c}5 \\
23.8 \%\end{array}$ & $\begin{array}{c}1 \\
4.8 \%\end{array}$ & $\begin{array}{c}21 \\
23.8 \%\end{array}$ \\
\hline $\begin{array}{c}\text { Cerebral } \\
\text { Palsy }\end{array}$ & 0 & 0 & $\begin{array}{c}4 \\
100 \%\end{array}$ & 0 & $\begin{array}{c}4 \\
4.4 \%\end{array}$ \\
\hline Renal failure & $\begin{array}{c}1 \\
8.3 \%\end{array}$ & $\begin{array}{c}2 \\
16.7 \%\end{array}$ & $\begin{array}{c}6 \\
50 \%\end{array}$ & $\begin{array}{c}3 \\
25 \%\end{array}$ & $\begin{array}{c}12 \\
13.3 \%\end{array}$ \\
\hline Rh- HD & 0 & $\begin{array}{c}1 \\
12.5 \%\end{array}$ & $\begin{array}{c}7 \\
87.5 \%\end{array}$ & 0 & $\begin{array}{c}8 \\
8.8 \%\end{array}$ \\
\hline Sickle cell anemia & 0 & 0 & $\begin{array}{c}5 \\
71.4 \%\end{array}$ & $\begin{array}{c}2 \\
28.6 \%\end{array}$ & $\begin{array}{c}7 \\
7.7 \%\end{array}$ \\
\hline $\begin{array}{c}\text { Thalassemia } \\
\text { major }\end{array}$ & 0 & 0 & $\begin{array}{c}5 \\
100 \%\end{array}$ & 0 & $\begin{array}{c}5 \\
5.5 \%\end{array}$ \\
\hline Total & $\begin{array}{c}28 \\
31.1 \%\end{array}$ & $\begin{array}{c}17 \\
18.9 \%\end{array}$ & $\begin{array}{c}38 \\
42.2 \%\end{array}$ & $\begin{array}{c}7 \\
7.8 \%\end{array}$ & $\begin{array}{c}90 \\
100 \%\end{array}$ \\
\hline
\end{tabular}


Amira Aly Salem, Mohamed Amr Hussein and Lobna Monsour .

Table (13): Frequency And Percent Distribution Of Patients According To Hemoglobin And Serum Albumin Levels ( $\mathrm{N}=150)$.

\begin{tabular}{|l|l|l|}
\hline Variable & No. & $\%$ \\
\hline Hemoglobin $\mathrm{g} \%$ & 84 & 50.6 \\
\hline$<10 \mathrm{~g}$ & 66 & 44.0 \\
\hline$>10 \mathrm{~g}$ & \multicolumn{2}{|l|}{} \\
\hline Serum albumin $\mathrm{g} \%$ & 23.4 \\
\hline$<2.5$ & 35 & 22.6 \\
\hline $2.5-3$ & 34 & 32.6 \\
\hline $3-3.5$ & 49 & 21.4 \\
\hline$>3.5$ & 32 & \\
\hline
\end{tabular}

Table (14): frequency and percent distribution of patients according total lymphocyte count.

\begin{tabular}{|l|l|l|}
\hline Total lymphocyte count Cells $/ \mathrm{mm}^{3}$ & No. & $\%$ \\
\hline$<500$ & 34 & 22.6 \\
\hline $500-1000$ & 25 & 16.6 \\
\hline $1000-1500$ & 50 & 33.3 \\
\hline$>1500$ & 41 & 27.5 \\
\hline
\end{tabular}




\section{References}

Bistarian BR, Blackburn GL, Vitalle J, Cochran D, Naglor J (1976):

Prevalence of malnutrition in general medical patients, J. Am. Med. Ascoc. 235:1567.

Commey- Jo; Amuasr. GA; Richardson JE, Asamoorh- Baah (1985).

The nutritional status \& feeding practices among sick urban preschool chitdren admitted to a major hospital in Accra, Ghana, Am- Trop. Pediatr 5(3): 131-6.

Cunningham - Rundles S and Cervia J.S (1997)

"Malnutrition and host defense" from Nutrition in Pediatrics. Textbook of pediatrics $2^{\text {nd }}$ ed.BC. Decker. Inc Publisher Hamilton chapter 19; P 295:307.

Hamill, PW; Drizd TA, Johnson Cl, Roch AF, Moore W(1979)

Physical growth : National Center for Health statistics, Percentiles, Am J. Clin. Nut. $32: 607-629$.

Herman FR, Safran C, Levkoff SE, Minaker KL (1992),

Serum albumin level on admission as a predictor of death, length of stay and readmission Arch. Intern. Med. 152:125-130.

Jelliffe DB (1966):

The assessment of nutritional status of the community. WHO Monograph series No. 53 , WHO, Geneva.

Karp RJ(ed) : (1993;)

Malnourished children in the United states, New York, Springer.

Keusch, GT (1990)

Malnutritoin infection and immune function in the malnourished child. Edited by RM Suskind \& LL Suskind Nestle Nutrition Workshop series Vol. 19 NY, Raven Press, P 37 39. 


\section{Amira Aly Salem, Mohamed Amr Hussein and Lobna Monsour .}

Kristy MH, Christopher D, Linda G, Alexandra c (1995)

Malnutrition in Hospitalized Pediatric Patients. Current prevalence, Arch. Pediatr. Adolesc. Med; 149:1118-1122.

Mc Laren D.S, Barman D, Belton NR, Williams AF (1991)

Textbook of Pediatric Nutrition, Churchill livingstone, Edinburgh.

Mc Whirter JP, Pennington CR (1994)

Incidence and recognition of malnutrition in hospital; British Medical Journal 308:945948.

Merritt RJ., Suskind RM (1979)

Nutritional Survey of hospitalized pediatric patients Am J. Clin. Nut. 32:1320-1325.

Merritt RJ, Kalsch M, Roux LD, Ashely, Mills J, Sigel S (1984);

Significance of hypo- albuminemia in pediatric oncology patients; malnutrition or infection. J Parenteral Enteral Nutr., 9:303-306.

Phillips A, Sharper AG, Whincup PH (1989)

Association between serum albumin \& mortality from cardio vascular disease cancer and other causes. Lancet II 1434-1436.

Waterlow JC (1972)

Classification of PEM depending on length / age and weight / age Br. Med., J., 2:556.

Waterlow JC (1974)

Some aspects of childhood malnutrition as a public health problem $\mathrm{Br}$. Med. $\mathrm{J}, 4: 88$.

Whitehead RG, Coward WA, Lunn PG (1973)

Serum albumin concentration and the onset of Kwashiorkor, Lancet, 1:63. 
معل إنتشار سنو التغذية لاى الاطفل عند دخولهم للعلاج بمستشفى الاطفلّ.

$$
\begin{aligned}
& \text { الميرة على سالم * ـ محمد عمرو حسين** ـ لبنى منصور * } \\
& \text { * قسم طب الاطفل لية ألطب - جامعة القاهرة . } \\
& \text {.المعه القومي للتنذية . } \\
& \text { الملخص العربي }
\end{aligned}
$$

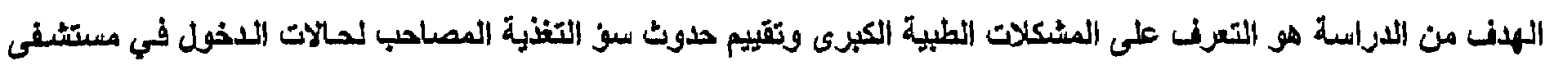

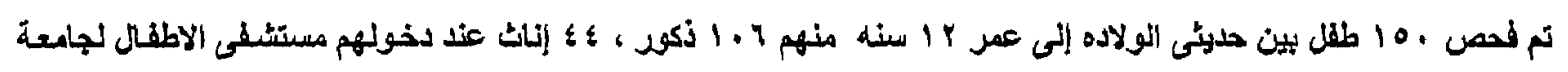

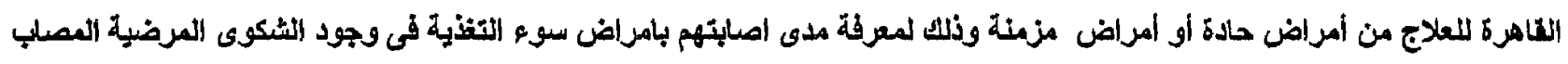

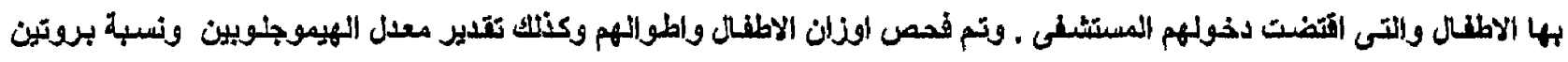

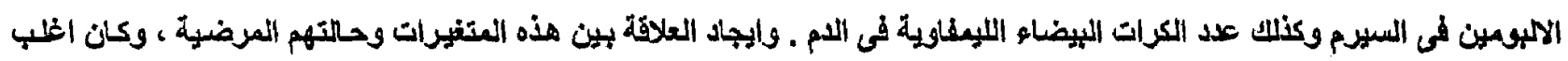

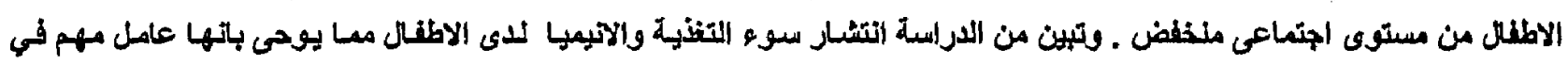

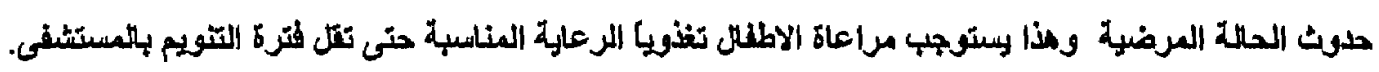

